\title{
Enzo V. Alliegro, Out of Place Out of Control. Antropologia dell'ambiente-in-crisi
}

CISU, Roma, 2020

Elena Bougleux

\section{OpenEdition}

\section{Journals}

Edizione digitale

URL: http://journals.openedition.org/aam/3582

DOI: $10.4000 /$ aam.3582

ISSN: 2038-3215

Editore

Dipartimento Culture e Società - Università di Palermo

Notizia bibliografica digitale

Elena Bougleux, «Enzo V. Alliegro, Out of Place Out of Control. Antropologia dell'ambiente-in-crisi», Archivio antropologico mediterraneo [Online], Anno XXIII, n. 22 (2) | 2020, online dal 31 décembre 2020 consultato il 30 janvier 2021. URL: http://journals.openedition.org/aam/3582 ; DOI: https://doi.org/ 10.4000/aam.3582

Questo documento è stato generato automaticamente il 30 janvier 2021.

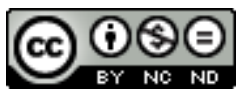

Archivio antropologico mediterraneo è distribuita con Licenza Creative Commons Attribuzione - Non commerciale - Non opere derivate 4.0 Internazionale. 


\section{Enzo V. Alliegro, Out of Place Out of Control. Antropologia dell'ambiente-in- crisi}

CISU, Roma, 2020

Elena Bougleux

NOTIZIA

ENZO V. ALLIEGRO, Out of Place Out of Control. Antropologia dell'ambiente-in-crisi, CISU, Roma, 2020.

ISBN 9788879757010 
1

Enzo Alliegro radica il suo accurato lavoro di ricerca all'interno della storia. La storia è la sua area intellettuale di riferimento, il suo principale serbatoio di informazioni, di narrazioni e di dati. Alliegro si confronta senza tentennamenti epistemologici anche con le tracce esili della storia recente, con la storia minuta, con il fatto contemporaneo e immeditato, utilizzando gli stessi strumenti, la stessa attenzione e lo stesso rigore con cui si confronta - in altri suoi studi, e in misura minore anche in questo - con gli archivi della Storia con la S maiuscola. Nel suo volume appena uscito per i tipi di CISU, in ogni capitolo di Out of Place Out of Control. Antropologia dell'ambiente-in-crisi, il suo serbatoio di storia è costituito dai materiali grigi dei verbali di infinte riunioni, dai report di documentazione istituzionale, dagli esposti presso le avvocature, a cui l'autore attribuisce la stessa dignità di fonti bibliotecarie antiche. I documenti ricavati dai consigli comunali, dalle cartelle delle ASL, dai comitati di cittadini mobilitati per la difesa dei propri diritti sono attraversati con la stessa pignoleria, aspettativa di esattezza e coerenza con cui si attraversano i database della grande scienza. Ma tali documenti, un po' per la loro intrinseca natura, molto invece per ragioni del tutto antropogeniche, quasi mai riescono a reggere il confronto con la disamina severa dell'autore: la coerenza latita, le contraddizioni e le omissioni ricorrono, le responsabilità emergono chiare come montagne.

2 In questa ricerca, Enzo Alliegro ricava dall'attenta analisi delle fonti micro-storiche una quantità sorprendente di (gravi) dati ambientali, di indizi di sofferenza presso (ampi) contesti sociali e (inquietanti) prossimità politiche, tutti elementi che convergono verso un unico esito: una fotografia amara di una parte di questo paese, il Sud, e del suo territorio, scandagliato sequenzialmente per regioni - la Basilicata, la Campania, la Puglia. Una fotografia amara che certamente si concentra sul presente, ma che essendo fortemente sorretta dalle evidenze scritte non lascia spazio per una interpretazione effimera, circoscritta e limitata alla cronaca. Poter radicare una ricerca di antropologia del contemporaneo all'interno di fonti scritte, a loro volta contemporanee, e sostenere fortemente l'etnografia - strumento a volte fragile - significa intraprendere quella che l'autore chiama una ricerca multimodale, che vive del sincretismo delle fonti. Nel corso dei suoi multimodali accessi alle fonti, Alliegro non esita a confrontarsi con i dati quantitativi, con la chimica industriale, con la strumentazione tecnologica, e questo gli permette di associare alla condizione di crisi ambientale una serie di cause profonde e lontane, non locali né contingenti; una visione ampia che forse gli permette di suggerire dove andare a cercare (l'inizio di) possibili cure. Questa è una delle missioni non da poco che si prefigge questo volume, che non si limita dunque ad essere una denuncia delle crisi ambientali del presente in Sud Italia. 
Out of Place Out of Control è ovviamente un volume fortemente antropologico, come ci annuncia il suo sottotitolo con le lineette, Antropologia dell'ambiente-in-crisi, perché le storie degli ambienti-in-crisi sono analizzate e raccontate come inseparabili da quelle delle persone, singoli soggetti e intere comunità, in una relazione ciclica di reciproca dipendenza e distruzione, impossibile da interrompere. Il rapporto (troppo) stretto tra le comunità e i loro territori offesi dai fumi delle raffinerie, dalle polveri dei metalli e dal gas radon (anzi gass-ra-doon) lega gli attori locali e gli scenari in una rete multipla, consapevolmente dannosa, fatta di speranza, memoria, puntuale disillusione, lotta, malattia, di nuovo speranza. Una relazione che non finisce anzi diventa sempre più densa di simboli e di materia.

Out of Place Out of Control è un volume che parla di natura: di natura semplice, nei suoi elementi fondamentali, l'aria, la terra, l'acqua e il fuoco (ci sono proprio tutti), ma anche di natura elaborata e costruita, nei suoi elementi evoluti e composti, il petrolio, l'acciaio, le plastiche, quegli agglomerati indistinti di scarti della modernità, nature iper-processate, che alimentano gli incendi della Terra dei Fuochi. È un libro quindi su molte nature: a seconda dei casi nature trasformate, sfruttate, complessificate, riadattate, e poi anche scartate, inalate, assorbite, sofferte. In questo processo di continua e accelerata trasformazione di nature, gli elementi primari e semplici finiscono sullo sfondo: ci sono ancora, ma non sono più riconoscibili né possono svolgere le loro fondamentali funzioni. L'aria non è più la stessa aria, l'acqua non è più la stessa acqua, entrambe sono diventate fonti di avvelenamento, cause di patologie, marker evidenti della fine dell'appaesamento. Nonostante le comunità qui raccontate non si spostino mai dai propri territori di origine, e anzi al contrario, i più agguerriti tra gli attivisti ambientali siano proprio quelli che rivendicano con più forza l'appartenenza, che non se ne sono mai andati e non si rassegnano a farlo, una forte crisi della presenza permea tutti i capitoli legati all'attivismo: in che senso si può 'appartenere a un luogo', quando il luogo non ha più la stessa faccia, né odore né sapore, e al contrario, abbassa l'aspettativa di vita di chi vi risiede? Credo che l'out of Control del titolo del volume di Enzo Alliegro si possa riferire anche a questo aspetto: fuori dal controllo sono le emozioni e le sensazioni di appartenenza, che in questi contesti da cui si vorrebbe scappare dettano invece un comportamento di resistenza e attaccamento più forte di ogni controllo razionale.

5 Per parlare di crisi della presenza l'autore passa da Heidegger e da de Martino, analizza l'immanenza e la durata - perché questo autore ci ha abituato a seguirlo in un spoletta continua tra minimo dettaglio etnografico e astratta concettualizzazione, con uno stile molto ricco e personale che si nutre di molti neologismi - ma poi si ferma in piazza Masaccio nel quartiere Tamburi e sui binari di San Pietro Vernotico, dove una parte importante della sua ricerca multisituata si interrompe all'improvviso, per opera della forza pubblica, e poi della magistratura. 'Multimodale' è un termine appena didascalico, quasi riduttivo, per descrivere quello che l'autore intende come suo metodo di lavoro.

Leggendo in un solo sguardo l'Ambiente-in-crisi, come ci chiede di fare l'autore, viene fatto di pensare che l'ambiente in crisi sia l'oggetto della sua antropologia, e che questo oggetto sia diverso dall'ambiente tout court. Dal testo direi invece che emerge come tutti gli ambienti siano ambienti-in-crisi, e anche di più, che ad occuparsi dell'ambiente-in-crisi sia proprio tutta l'antropologia: non è possibile infatti affrontare l'antropologia dell'ambiente senza incontrarsi con l'antropologia medica, l'antropologia urbana, con quella delle organizzazioni e con lo studio movimenti 
sociali, con la environmental justice e con la citizen science, non per diventare anglofili ma solo per evidenziare come appena fuori dai nostri confini anche queste linee di ricerca per noi ancora un po' marginali siano invece ben diffuse e affermate. Inoltre, anche se nel volume viene appena rivelato, va sottolineato come l'antropologo vada-in-crisi insieme alla sua antropologia, se decide di occuparsi di ambiente; non solo in senso riflessivo, ma anche perché più precisamente, l'antropologo va in crisi a causa della sua antropologia dell'ambiente. Manipolare con disinvoltura i materiali grigi di cui abbiamo parlato all'inizio significa gestire una materia incandescente, densa di nomi e cognomi, di riferimenti esatti e non derogabili nella loro evidenza - pena la perdita di credibilità degli stessi fatti riferiti.

7 L'antropologia dell'ambiente-in-crisi non fa sconti metodologici all'autore, che nel caso di questo volume affronta consapevolmente i rischi della rinuncia alla copertura delle proprie fonti e percorsi, perché, come ci dice lui stesso, la crisi ambientale fa emergere una sequenza complessa di problemi sociali, l'ambiente al contrario di ogni visione bucolica è un catalizzatore di crisi, e le crisi vanno raccontate esattamente come sono, con il loro esatto nome. La crisi che qui emerge più chiara è quella del Mezzogiorno, che usando le parole dell'autore, è la cartina di tornasole della crisi della modernità. Vaste aree meridionali economicamente depresse vivono una condizione di crisi del moderno senza mai essere state moderne, avendo attraversato la modernità (questa modernità, petrolifera, industriale e iper-metropolitana) solo a parole. Una condizione che ci ricorda altre aree del pianeta, come il sud dell'India o alcune zone dell'America Latina, dove la condizione di arretratezza è diventata elemento strutturale dell'epoca postmoderna, ed è stata introiettata a livello identitario in un modo che sembra permanente, non più riconducibile ad una singola e specifica crisi. Nei confronti di queste aree del pianeta l'economia egemonica del capitale ha giocato lo stesso ruolo che nel Meridione italiano hanno giocato alcuni poteri economici forti, talvolta affiancati $\mathrm{da}$ ambiti più o meno riconoscibili della illegalità organizzata - e qui naturalmente mi riferisco al bel capitolo del volume dedicato al ciclo dei rifiuti in Campania. Il problema dell'interiorizzazione identitaria della condizione di crisi, riferita allo spazio vitale e di riflesso ai soggetti, crea per l'autore la necessità di introdurre uno dei concetti chiave per leggere tutto il volume, il concetto di etnostrabismo. Il dispositivo etnostrabico ha il potere di nascondere alla vista e al giudizio degli attori sociali (ma a volte anche degli studiosi) una assoluta evidenza, che resta nascosta dentro le pieghe di un pezzo di realtà che ha preso il sopravvento narrativo, immaginifico, estetico, e che diventa totalizzante, restando l'unica realtà. La maggior parte delle crisi dell'ambiente - direi meglio, delle loro cause prime - sono nascoste proprio da meccanismi etnostrabici, che hanno traslato l'attenzione sui dettagli, e lasciato la scena complessiva fuori fuoco, rendendoci appunto incapaci di cogliere delle (future) evidenze. L'ambiente con suoi meccanismi antichi e complessi è un soggetto (sì, voglio dire soggetto) resiliente, reagisce poco e lentamente alle pressioni e aggressioni antropiche, rendendo l'etnostrabismo non solo possibile (è facile non vedere) ma anche duraturo (per lungo tempo non succede niente di evidente). Quando però la resilienza cessa e la crisi esplode, il dispositivo etnostrabico crolla all'improvviso, e il sistema-mondo collassa per intero, dalla base: dunque tutte insieme, crisi della presenza, ma anche del lavoro, della salute, della stessa possibilità di restare-abitare.

8 Per cogliere questo stato di volontario obnubilamento della comprensione, che si tende a far durare più a lungo possibile, il tempo di una caduca ma accanita autodifesa, Alliegro usa un'altra espressione feconda, memorial divide, intendendo una sorta di 
malattia selettiva della memoria che coglie gli attori, e di nuovo, anche gli studiosi dell'ambiente-in-crisi. A differenza di altri campi di studio a noi vicini, come appunto la storia e anche le scienze esatte, che costruiscono i passi nuovi dei propri saperi sulle stratificazioni dense dei propri saperi sedimentati, l'antropologia dell'ambiente-in-crisi dimentica, dimentica quasi tutto, rimuove e poi con grande fatica ricostruisce, ogni volta dovendo riscoprire e comprendere i problemi da capo. Le dimenticanze sono selettive, mirate, colpevoli: osservate a posteriori appaiono quasi deliberate e volontarie.

Il meccanismo di superamento del memorial divide va messo a punto con grande cautela e scrupolo, e questo volume fornisce una pista possibile da seguire, inondando di dati esatti le sue pagine e le sue note. Ma forse non basta, i dati e soprattutto le note sono state ben presenti in letteratura, e sono finite dimenticate soprattutto le note, oppure saltate ad una lettura veloce, quasi l'unica rimasta possibile, tristemente. Per fortuna c'è anche una pista diversa, amata dall'autore, ed è quella simbolica. Alliegro è uno studioso dei simboli e dell'architettura dei loro significati, e qui la sua storia di studioso della tradizione entra in gioco in modo dialettico: simbolo non è necessariamente antico, simbolo non ci arriva necessariamente dalla tradizione, piuttosto il simbolo emerge come coagulo di sensi dissonanti, come sintesi semantica imprevista e molto presto autonoma dai suoi artefici. Nella descrizione della crisi delle scuole di Taranto, le cui finestre devono restare al tempo stesso aperte e chiuse - aperte per far uscire il gas radon, tossico, esalato dal suolo e infiltrato negli edifici, e chiuse, per non far entrare i fumi dell'Ilva e i micro detriti volatili rilasciati dalle collinette di scarti industriali nei giorni di vento (curiosamente chiamati nei report wind days, e non giorni di vento) - il gas stesso emerge come potente simbolo. Il gas, i gas, i fumi, il respirabile, quello che resta dell'aria come elemento semplice, sono al tempo stesso potente simbolo e ordinaria materia, condensato astratto di significati culturali e oggetto tangibile. I gas poi, nella loro invisibilità, onnipresenza, in certi casi apparente inafferrabilità, non appaiono così tangibili e per questo si prestano ancora meglio al ruolo di aggregatore simbolico. Il fuoco che esala dalla Terra dei Fuochi è materico e simbolico, il petrolio della Valle dell'Agri è materico e simbolico. Dalle parole dell'autore deduciamo che non si possono condurre studi sull'ambiente senza ricorrere ai simboli, che sintetizzano la crisi e indicano la via per il superamento del memorial divide - perché i simboli sono fuori dal tempo, e a differenza della materia sopravvivono alle crisi. Quindi, per estensione, non si possono condurre studi sull'ambiente senza ricorrere all'antropologia.

Enzo Alliegro dunque conia le parole e poi le articola, le combina e ricombina, dimostrando il suo ragionamento sottile a partire dai suoi propri termini: talvolta è difficile seguirlo, ma quando finalmente lo hai seguito è poi difficile dargli torto, perché questo andrebbe fatto usando i suoi stessi nomi e i suoi concetti, usando i quali però si resta presi nella sua rete di significati. In tal senso la lettura di questo autore è anche un proficuo esercizio intellettuale, una palestra di ragionamento costruttivo-induttivo essenziale per le menti antropologiche (e non solo). Ancora una volta, un valido motivo per cimentarsi in questa lettura.

11 Ma mentre l'autore concludeva il volume, il Covid-19 - un evidente e ulteriore segnale di ambiente-in-crisi, è diventato il protagonista del presente, ha occupato le prime file di tutti gli scenari della pratica privata e del discorso pubblico, gettando nell'ombra tutte le altre crisi, che non si sono affatto fermate, al contrario, ma hanno smesso di 
essere guardate e raccontate. Il capitolo conclusivo del libro è dunque una prova di abilità dell'autore, che applica in tempo reale ad un problema ambientale del tutto nuovo il suo arsenale metodologico e linguistico sviluppato per altri terreni (ma in realtà per dirla tutta eravamo stati un po' etnostrabici anche noi, dato che i problemi derivanti dallo spill-over - passaggio virale tra specie eterogenee - e le pandemie di origine animale non erano proprio nuovi).

L'autore nelle ultime pagine ci consegna così una interessante e anche intrigante lettura etnografico-simbolica-a-distanza del mercato degli animali vivi di Wuhan, come un luogo umido, buio e pericoloso, affollatissimo, antico e ipermoderno al tempo stesso, il luogo dell'inizio di tutto, forse. Si tratta della palestra perfetta entro cui Enzo Alliegro mette alla prova i suoi strumenti di analisi concettuale e fattuale, articolando domande analoghe a quelle elaborate in anni di campo: come facciamo ad avere paura del luogo più familiare, quello dove si compra il cibo (crisi della presenza), come possiamo controllare un virus che viaggia in aereo per il globo all'insaputa dei suoi ospiti (entità invisibile simbolico-materica), come ci possiamo difendere da qualcosa che ora abbiamo in casa (auto-identificazione con lo stato di crisi). Veramente un bel libro, che andrebbe letto attentamente anche da chi non è antropologo e non si occupa (crede di non occuparsi) dell'ambiente in crisi. 